\title{
Antineoplastic effects of an Aurora B kinase inhibitor in breast cancer
}

\author{
Christopher P Gully ${ }^{1,2,3}$, Fanmao Zhang ${ }^{1,2}$, Jian Chen ${ }^{1}$, James A Yeung ${ }^{1}$, Guermarie Velazquez-Torres ${ }^{1,2,6}$, \\ Edward Wang ${ }^{1,2,6}$, Sai-Ching Jim Yeung ${ }^{4,5^{*}}$, Mong-Hong Lee Le, $^{1,23^{*}}$
}

\begin{abstract}
Background: Aurora B kinase is an important mitotic kinase involved in chromosome segregation and cytokinesis. It is overexpressed in many cancers and thus may be an important molecular target for chemotherapy. AZD1152 is the prodrug for AZD1152-HQPA, which is a selective inhibitor of Aurora B kinase activity. Preclinical antineoplastic activity of AZD1152 against acute myelogenous leukemia, multiple myeloma and colorectal cancer has been reported. However, this compound has not been evaluated in breast cancer, the second leading cause of cancer deaths among women.

Results: The antineoplastic activity of AZD1152-HQPA in six human breast cancer cell lines, three of which overexpress HER2, is demonstrated. AZD1152-HQPA specifically inhibited Aurora B kinase activity in breast cancer cells, thereby causing mitotic catastrophe, polyploidy and apoptosis, which in turn led to apoptotic death. AZD1152 administration efficiently suppressed the tumor growth in a breast cancer cell xenograft model. In addition, AZD1152 also inhibited pulmonary metastatic nodule formation in a metastatic breast cancer model. Notably, it was also found that the protein level of Aurora B kinase declined after inhibition of Aurora B kinase activity by AZD1152-HQPA in a time- and dose-dependent manner. Investigation of the underlying mechanism suggested that AZD1152-HQPA accelerated protein turnover of Aurora B via enhancing its ubiquitination.
\end{abstract}

Conclusions: It was shown that AZD1152 is an effective antineoplastic agent for breast cancer, and our results define a novel mechanism for posttranscriptional regulation of Aurora B after AZD1152 treatment and provide insight into dosing regimen design for this kinase inhibitor in metastatic breast cancer treatment.

\section{Background}

Aurora kinases are a family of serine/threonine kinases which share $\sim 70 \%$ homology in their kinase domains [1-3], and they are essential in cell cycle control and mitosis [3-6]. Mammalian cells have three Aurora family members: A, B and C. The Aurora kinases are expressed at maximum levels during mitosis. Aurora A and B have different subcellular localizations and serve distinct functions during mitosis. Together, they tightly manage chromosome segregation to ensure that each resulting daughter cell receives a full complement of chromosomes [3-5]. All three Aurora kinases have been shown

\footnotetext{
* Correspondence: syeung@mdanderson.org; mhlee@mdanderson.org
${ }^{1}$ Department of Molecular and Cellular Oncology, The University of Texas

* Correspondence: syeung@mdanderson.org; mhlee@mdanderson.org
${ }^{1}$ Department of Molecular and Cellular Oncology, The University of Texas MD Anderson Cancer Center, Houston, TX 77030, USA
${ }^{4}$ Department of General Internal Medicine, Ambulatory Treatment \& MD Anderson Cancer Center, Houston, TX 77030, USA
${ }^{4}$ Department of General Internal Medicine, Ambulatory Treatment \& Emergency Care, The University of Texas MD Anderson Cancer Center, Houston, TX 77030, USA
}

(c) 2010 Gully et al; licensee BioMed Central Ltd. This is an Open Access article distributed under the terms of the Creative Commons Attribution License (http://creativecommons.org/licenses/by/2.0), which permits unrestricted use, distribution, and reproduction in any medium, provided the original work is properly cited. contribute to tumorigenesis through their link to invasive disease and genomic instability [3]. By virtue of their important role in cell proliferation and their oncogenic potential [7], the Aurora kinases are potentially important targets for cancer therapeutics.

In contrast to Aurora A which localizes to the centrosomes and contributes to spindle bi-polarity by managing microtubule assembly and centrosome organization, Aurora B maintains correct kinetochoremicrotubule attachments and is localized to the chromosomes during metaphase. Aurora B relocalizes to the midbody of the cell during late anaphase and telophase, which hints at an additional function during cytokinesis [4]. Aurora B phosphorylates Histone $\mathrm{H} 3$ at serine 10 leading to dissociation of Histone $\mathrm{H} 1$ and chromatin condensation [8]. As a regulator of chromosome segregation, Aurora B is part of the Chromosome Passenger 
Complex (CPC) which includes its substrates: INCENP, Borealin and Survivin. The CPC governs the spindle checkpoint and manages correct microtubule attachments to kinetochores [6-9].

Aurora $\mathrm{B}$ has been demonstrated to be overexpressed in multiple myeloma [10], AML [1], colorectal [7], prostate [11] and pancreatic [12] cancers. In human breast cancer, an oncogenic link to Aurora B has not been made although Aurora A may be overexpressed in 95\% of cases [13] and may be used as a predictor of survival [14]. Overexpression of Aurora A may not simply be a gain of oncogenic function, rather Aurora A may be interfering with the delicate balance of Aurora B in the cell [15]. Aurora A kinase activating mutations do not further enhance the transformation phenotype of Aurora A [16]. Inhibition kinase activities of both Aurora A and B by ZM447439, a pan Aurora kinase inhibitor, results in cellular changes that most resemble loss of Aurora B function [15], and mutations in Aurora B confer resistance of HCT116 cells to ZM447439 [17]. Therefore, Aurora B may in fact be a more important drug target than Aurora A.

In this study, the knowledge gap regarding the use of the Aurora B-specific inhibitor, AZD1152, in breast cancer is addressed. AZD1152 is a dihydrogen phosphate prodrug and is metabolized in the serum to its active form, AZD1152-HQPA [hydroxyquinazoline pyrazol anilide], which is a small molecule ATP binding pocket competitor [18]. AZD1152-HQPA has potent selectivity for inhibition of Aurora $B\left[K_{i}=0.36 \mathrm{nM}\right]$ compared with Aurora $A\left[K_{i}=1,369 n M\right]$ and a panel of 50 other kinases [7]. The antineoplastic effect of this drug has been demonstrated in human cancer cell lines, including colon, lung, and cervix [7], as well as leukemia cell lines and primary acute myeloid leukemia cultures [1].

Also evaluated were the dose-responses of AZD1152HQPA in 6 human breast cancer cell lines as well as the cellular consequences of Aurora B inhibition. Further, the antineoplastic effects of AZD1152 in nude xenograft mice using two breast cancer cell lines were examined. This was followed by the novel discovery that this Aurora B kinase inhibitor downregulated Aurora B protein level by increasing polyubiquitination and proteasomal degradation of Aurora B. Together, these studies indicate that AZD1152 has antineoplastic activity in human breast cancer cells and that AZD1152's impact on Aurora B protein stability is another important layer of regulation that has not been characterized before.

\section{Results}

Breast cancer cells are sensitive to AZD1152-HQPA in vitro and display signs of mitotic catastrophe after exposure

To evaluate the effect of AZD1152-HQPA on breast cancer cells, HER18 (ER+, PR+, p53wt) breast cancer cells, which stably overexpress HER2 (parent line, MCF-7) [19], were treated with AZD1152-HQPA. Cell proliferation was measured by MTT assay (Figure 1). The concentration that achieves $50 \%$ of maximal inhibition of proliferation $\left(\mathrm{IC}_{50}\right)$ of the cells was measured at $20 \mathrm{nM}$ by sigmoidal curve fitting. Similar results were obtained in MDA-MB-468 (HER2+, EGFR+++, Pten-, ER-, PR-, p53 mutant), MDA-MB-435 (HER2+[16], ER-, PR-, p53 mutant), MDA-MB-231 (HER2+[16], ER-, PR-, p53 mutant), MDA-MB-361 (HER2+++, ER+, PR+, p53wt) and BT-474 (HER2+++[14], ER+, PR+, p53 mutant) at $\mathrm{IC}_{50}$ of $14 \mathrm{nM}, 125 \mathrm{nM}, 105 \mathrm{nM}, 70 \mathrm{nM}$ and $8 \mathrm{nM}$ respectively. All human breast cancer cell lines tested showed sensitivity to AZD1152-HQPA with typical sigmoidal log (dose)-response curves. The observed $\mathrm{IC}_{50} \mathrm{~S}$ are comparable to those found in leukemia and other human cancer cell lines $[1,7]$. To verify the response to drug inhibition by MTT assay, a separate dose-response assay in HER18 cells measuring proliferation inhibition by live cell count, which corroborated the $\mathrm{IC}_{50}$ of $20 \mathrm{nM}$ for HER18 cells (data not shown), was performed. These results suggest that AZD1152-HQPA is an effective inhibitor of human breast cancer cells by MTT assay, a measure of both cell proliferation and cell death. Further, this effect was seen in various cell lines with multiple molecular profiles for HER2, ER, PR and p53.

\section{Treatment with AZD1152 causes mitotic catastrophe, G2/M arrest and polyploidy/aneuploidy}

Given the important role of Aurora B kinase in mitosis, the impact of AZD1152-HQPA on chromosome segregation was investigated. HER18 cells were incubated with or without $20 \mathrm{nM}$ AZD1152-HQPA for 48 hours. Chromosomal DNA was stained with DAPI and mitotic cells were observed by fluorescence microscopy. While control HER18 cells showed normal morphology (Figure 2A), cells treated with AZD1152-HQPA demonstrated characteristics of mitotic catastrophe including multinucleation, micronuclei and chromosome bridges (arrows and arrow heads). Quantification of this effect was performed and it was found that approximately $60 \%$ of drug-treated cells exhibited signs of mitotic catastrophe versus 3\% for control cells (Figure 2B). These observations are consistent with the effects of loss of Aurora B function and demonstrate that AZD1152HQPA is effective in causing mitotic catastrophe in breast cancer cells.

Measurement of the DNA content of individual HER18 cells treated with AZD1152-HQPA using flow cytometry after propidium iodide (PI) staining of DNA revealed that the percentage of $4 \mathrm{~N}$ HER18 cells increased after treatment with AZD1152-HQPA $20 \mathrm{nM}$ (Figure 2C). Importantly, HER18 cells with DNA content greater than $4 \mathrm{~N}$ began to appear after 48 hours of 


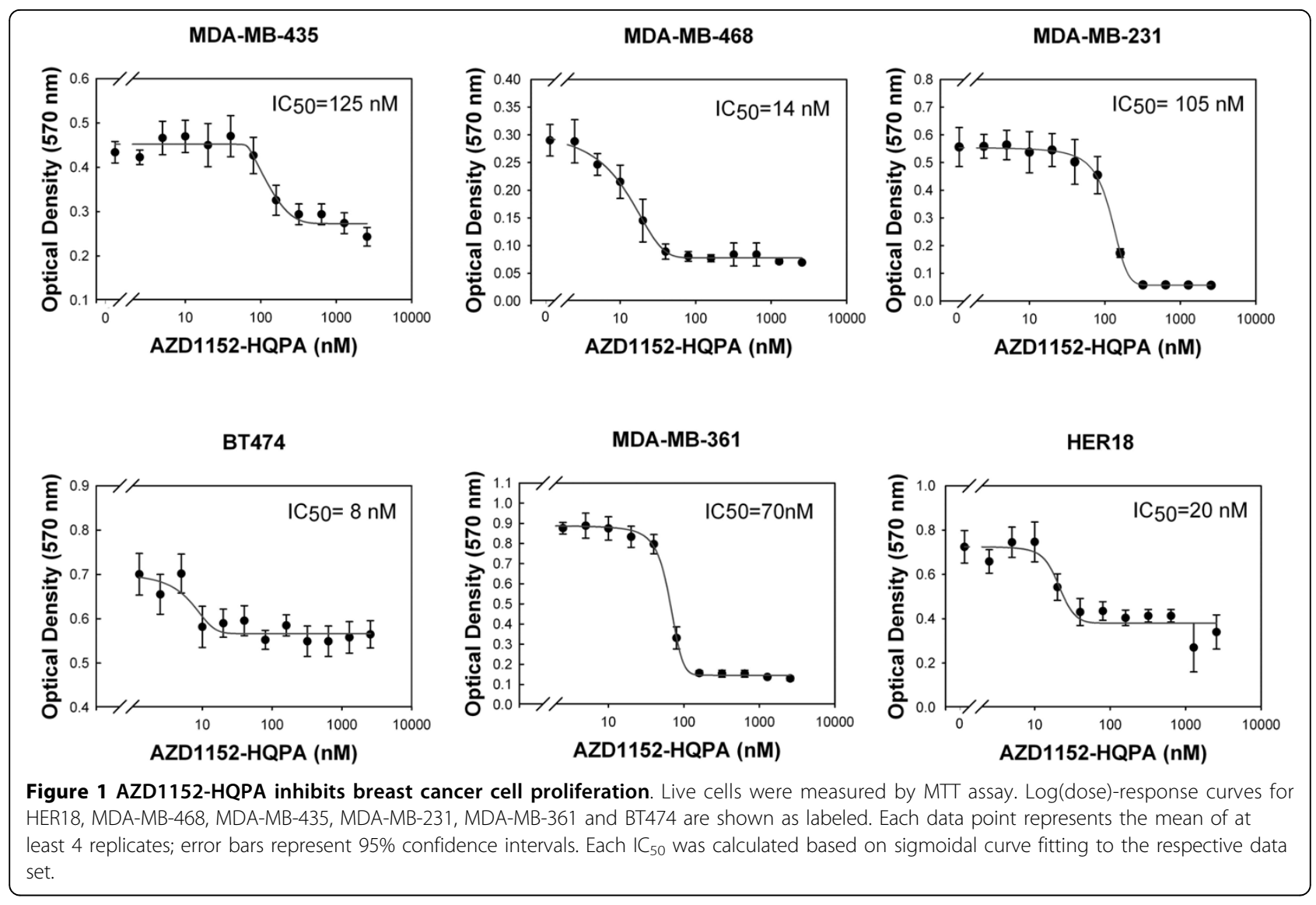

exposure to AZD1152-HQPA. The percentages of $2 \mathrm{~N}$, $4 \mathrm{~N}$ and $8 \mathrm{~N}$ cells were quantified from the flow cytometry data and are displayed in Figure 2D. It can be seen that as $G 2 / \mathrm{M}(4 \mathrm{~N})$ cell percentages increase, there is a concomitant decrease in G1 (2N) cell percentages. Polyploid $(8 \mathrm{~N})$ cells increased from $1.35 \%$ of the cell population at 24 hours to $9.7 \%$ at 48 hours. In Figure 2E, polyploid cell numbers were analyzed relative to the number of $8 \mathrm{~N}$ cells at time 0 . By 48 hours, the number of $8 \mathrm{~N}$ cells had increased 12.8 fold. This evidence, together with the observation of nuclear morphologic changes reported above, indicates that cells become polyploid or aneuploid as mitotic catastrophe occurs after treatment with AZD1152-HQPA.

\section{AZD1152-HQPA causes apoptosis and decreases clonogenic potential in breast cancer cells}

Next, AZD1152-HQPA treatment as the cause of cell death via apoptosis was investigated. HER18 cells were treated with AZD1152-HQPA (100 nM) for up to 48 hours, followed by staining with Annexin V-FITC/PI and analysis by flow cytometry (Figure 3A, upper panels). Percentages of cells in both early (lower right panel) and late (upper right panel) apoptosis increased with increasing duration of exposure to AZD1152-
HQPA. After 24 hours of treatment, there were 9.22\% apoptotic cells and $15.57 \%$ at 48 hours, compared to $5.64 \%$ in the untreated control cells (Figure 3A). Similar results were obtained with the parent cell line MCF7 (data not shown) as well as MDA-MB-231 (Figure 3A, lower panels). Apoptosis following AZD1152-HQPA treatment was also examined by immunoblotting to detect specific PARP (Poly [ADP-Ribose] Polymerase) cleavage by Caspase- 3 in HER 18 and MDA-MB-231 cells (Figure 3B). Increases in cleaved PARP in both cell types treated with AZD1152-HQPA were observed. This corresponds with the $\mathrm{IC}_{50}$ for these cell lines (Figure 1) and these results confirm that treatment with AZD1152-HQPA leads to apoptosis.

To determine if AZD1152-HQPA can inhibit the colony forming potential of breast cancer cells, HER18 cells were incubated at a low density with either control culture medium or $40 \mathrm{nM}$ AZD1152HQPA. After 12 days, colonies were stained with crystal violet and counted (Figure 3C). AZD1152HQPA inhibited the ability of HER18 cells to form colonies (Figure $3 \mathrm{C}$ and $3 \mathrm{D}$ ). The difference in the mean colony numbers per $\mathrm{cm}^{2}$ between the control and AZD1152-HQPA groups was significant ( $<<$ 0.001, two-sided Student's t-test). Next, the ability of 

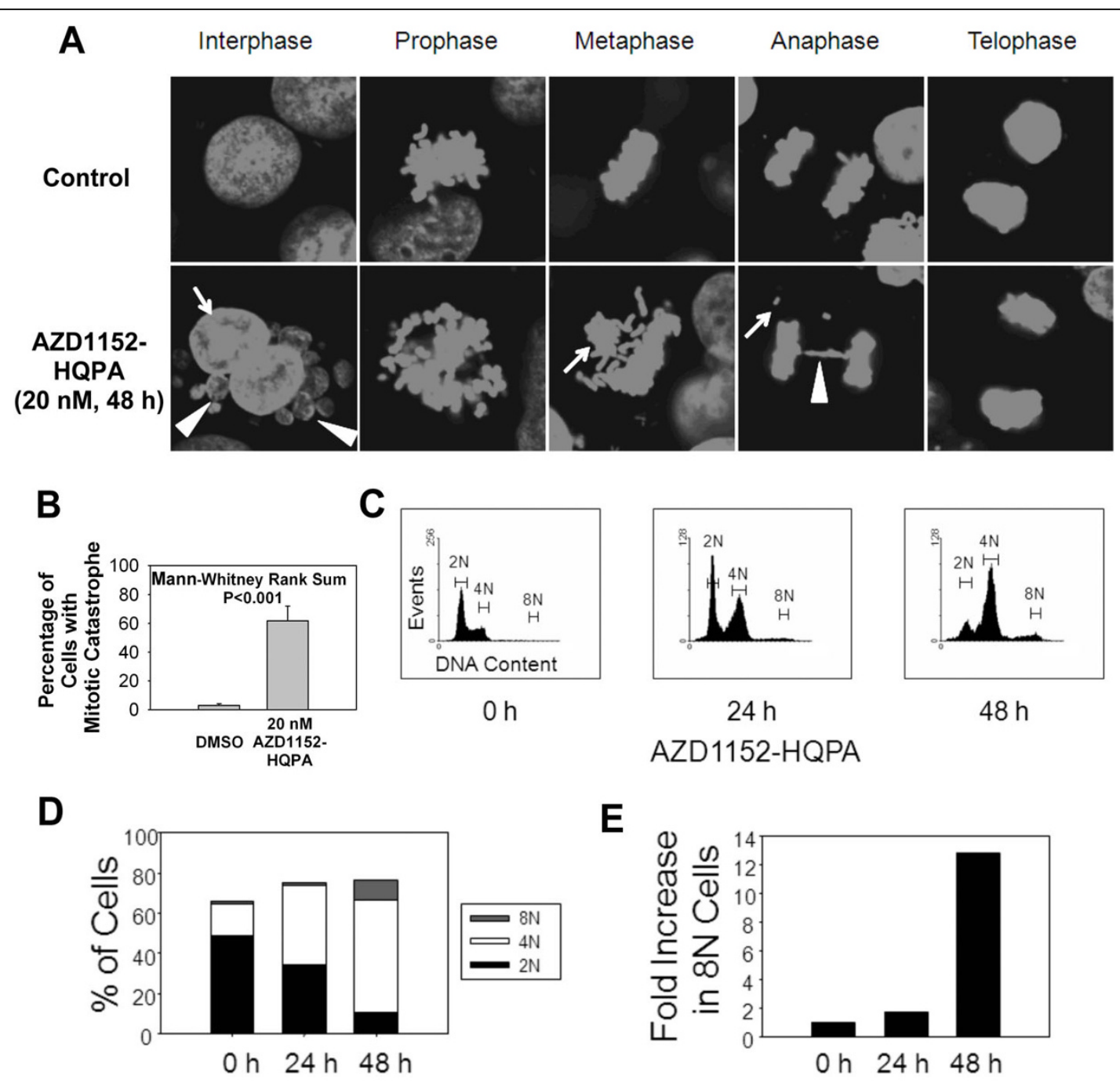

Figure 2 AZD1152-HQPA causes mitotic defects, aneuploidy and polyploidy in breast cancer cells. (A) HER18 cells were treated with control culture medium or $20 \mathrm{nM}$ AZD1152-HQPA for 48 hours, stained with DAPI and examined using fluorescence microscopy. Interphase cells showed bi-nucleation (arrow) and micronuclei (arrow heads). Metaphase cells showed misaligned chromosomes (arrow). Anaphase cells showed misegregated chromosomes (arrow) and chromosomal bridges (arrow head). (B) Percentage of cells from A with mitotic catastrophe is plotted for vehicle and 20 nM AZD1152-HQPA treatments. (C) HER18 cells were treated with AZD1152-HQPA (100 nM) for indicated times and stained with propidium iodide prior to analysis of DNA content by flow cytometry. Gating indicates $2 \mathrm{~N}(\mathrm{G} 1), 4 \mathrm{~N}(\mathrm{G} 2 / \mathrm{M})$ and $8 \mathrm{~N}$ (polyploid) cells. Aneuploid cells were present between $4 \mathrm{~N}$ and $8 \mathrm{~N}$ after $48 \mathrm{~h}$ treatment. (D) Percentages of $2 \mathrm{~N}, 4 \mathrm{~N}$ and $8 \mathrm{~N}$ cells are plotted at 0,24 and 48 hour time points based on the data in C. (E) Number of $8 \mathrm{~N}$ cells relative to 0 hours.

AZD1152-HQPA to inhibit anchorage independent growth of breast cancer cells was examined by using the soft agar colony forming assay. The mean colony number in soft agar was also significantly $(\mathrm{P}<0.001$, two-sided Student's t-test) decreased by $80 \mathrm{nM}$ AZD1152-HQPA compared with the control in both (Figure 3E).

Taken together, these results indicate that AZD1152HQPA has the ability to inhibit breast cancer cells by inducing apoptosis and decreasing their colony forming potential in both anchorage dependent and independent environments.
Aurora B inhibition by AZD1152 inhibits tumor growth in an orthotopic xenograft nude mouse model

To evaluate the in vivo effect of AZD1152 against aggressive breast cancer, HER18 human breast cancer cells were xenografted orthotopically into nude mice. 8.5 $\times 10^{6}$ HER18 cells were injected into the mammary fat pad of each female athymic $n u / n u$ mice that received weekly subcutaneous estradiol cypionate injections (3 mg/kg/dose) [20,21] starting 2 weeks prior to injection of tumor cells. When tumors were measurable (approximately $50 \mathrm{~mm}^{3}$, see Figure $4 \mathrm{~A}$ ), the mice were randomized into 3 groups: control $(n=6)$ which 


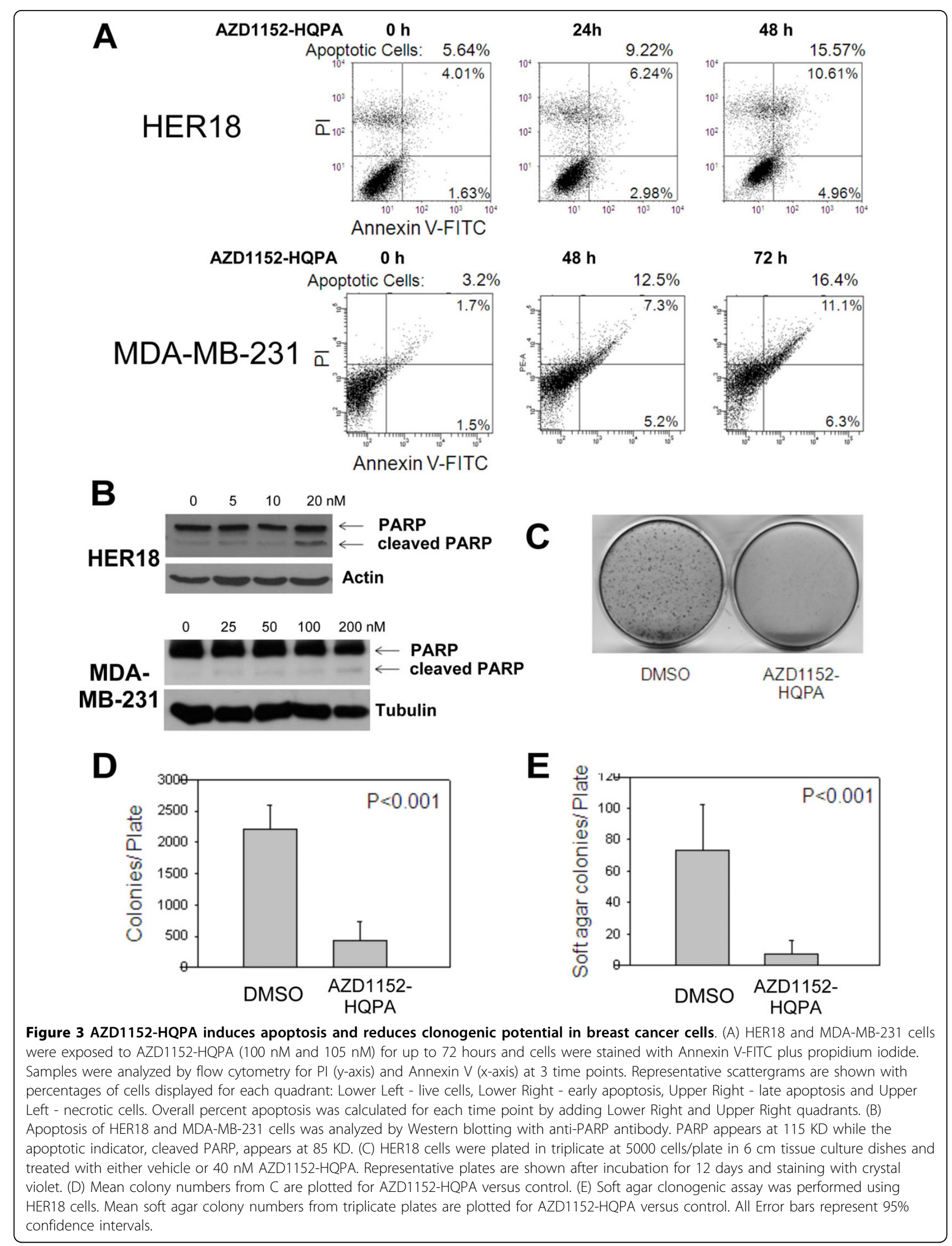


received $200 \mu \mathrm{l}$ of $0.3 \mathrm{M}$ Tris $\mathrm{pH} 9.0$, low dose $(\mathrm{n}=8)$ which received $62.5 \mathrm{mg} / \mathrm{kg} /$ dose AZD1152, and high dose $(\mathrm{n}=6)$ which received $125 \mathrm{mg} / \mathrm{kg} /$ dose AZD1152. Doses of AZD1152 were based on the pharmacokinetic findings of previous publications that a dose of 10-150 $\mathrm{mg} / \mathrm{kg} /$ day produced sufficient plasma concentrations of AZD1152 in nude mice $[7,18]$. Injections were administered IP on days 1 and 2 of a 7-day repeating cycle for 3 cycles. The mice treated with high dose AZD1152 showed reduced tumor volume compared to control mice $(\mathrm{P}<0.001)$ and low dose treated mice showed a near-significant reduction $(\mathrm{P}<0.08)$ (Figure $4 \mathrm{~A}$ and $4 \mathrm{~B}$ ). Excised tumors in the drug-treated groups weighed significantly $(\mathrm{P}<0.01$, one-way ANOVA, post-hoc intergroup comparison using Tukey test) less than those in the control mice (Figure 4C).

Formalin-fixed tumor samples were embedded in paraffin, and sections were examined by microscopy. Consistent with the in vitro data from Figure 2, multinucleate cells were observed on the hematoxylinand eosin-stained (H\&E) histological slides of tumor samples from AZD1152-treated mice but not on the slides of tumors from control mice (Figure 4D). Tumor samples were also snap-frozen in liquid nitrogen, and proteins were subsequently extracted for immunoblotting. Aurora B is known to phosphorylate serine 10 of Histone $\mathrm{H} 3$ [22] to aid in chromatin condensation by dissociating Histone $\mathrm{H} 1$ from heterochromatin [23,24]. Therefore, inhibition of Aurora B kinase activity can be verified by immunoblotting with phospho-specific antibody to serine 10 of Histone $\mathrm{H} 3$ [8]. A reduction in phospho-Histone H3 in AZD1152-treated tumors compared with control tumors (Figure 4E) was found, confirming that AZD1152 inhibited Aurora B kinase activity in vivo (i.e., confirming the pharmacodynamic mechanism). Immunohistochemistry staining for Ki-67 (a cell proliferation marker) and cleaved Caspase 3 (a marker of apoptosis) revealed that Ki-67 was markedly reduced in both drug treated groups when compared with the control group and that Caspase 3 cleavage was increased in the drug-treated groups versus the control. This provides evidence that AZD1152 induced apoptosis in breast cancer cells and inhibited breast cancer cell proliferation in vivo (Figure 4F).

\section{AZD1152 inhibits breast cancer metastasis}

In the above orthotopic xenograft assay spontaneous metastasis of tumors in either control or treatment groups was not observed. To address the question of whether AZD1152 could block metastases of breast cancer as well as growth of primary tumors, a breast cancer xenograft model with lung metastasis potential was employed. MDA-MB-231 human breast cancer cells known to be highly metastatic were used in this experimental assay. Six to eight week old female athymic $n u / n u$ mice were injected via tail vein with $2 \times 10^{6}$ MDA-MB-231 human breast cancer cells. Mice were randomized into 2 groups: control and AZD1152. Treatment with vehicle or AZD1152 (125 mg/kg/dose) began 2 days after intravenous injection of breast cancer cells. Drug or vehicle were administered IP on days 1 and 2 of a 7-day repeating cycle for 4 weeks only. Ten weeks after injection of cancer cells, mice were sacrificed, and the lungs were removed and weighed. The mean weight of lung tissue per mouse in the control group was significantly $(\mathrm{P}<0.01$, two-tailed $\mathrm{t}$-test) higher than that in the AZD1152 group (Figure 5A). Further, the number of macroscopic tumor nodules was found to be significantly ( $\mathrm{P}<0.008$, two-tailed t-test) higher in the lungs of control mice compared with AZD1152-treated mice (Figure 5C). Gross anatomic appearance of matched lung lobes from representative mice is shown in Figure $5 \mathrm{~B}$. H\&E staining of lung tissues from control and AZD1152-treated mice showed reduction of tumor burden by AZD1152 at the microscopic level (Figure 5D). The data show that AZD1152 is effective in inhibiting the aggressive and highly metastatic phenotype of MDA-MB-231 human breast cancer cells in vivo.

\section{AZD1152 reduces Aurora B protein level by increasing polyubiquitination and degradation via the proteasome} Examination of the impact of AZD1152-HQPA on Aurora $\mathrm{B}$ by immunoblotting also revealed that AZD1152HQPA inhibits Aurora B kinase activity. This was reflected by a decrease in both phosphorylated Histone H3, and Aurora B protein level in a dose- and timedependent manner (Figure 6A). The time courses showed that inhibition of Aurora B kinase activity preceded the decline in Aurora B protein level (Figure 6A). The unexpected finding of a decrease in Aurora B protein level was further investigated by examining the impact of AZD1152-HQPA on the protein turnover rate of Aurora B. HER18 cells were treated with or without 20 nM AZD1152-HQPA for 48 hours, then in the presence of cycloheximide (CHX) for up to four additional hours. After immunoblotting to measure Aurora B protein level (Figure 6B), the Aurora B protein band was quantified by densitometry. Relative protein level was calculated as the ratio of the integrated optical density of the Aurora B relative to time 0. The Aurora B protein turnover rates were determined as the slope of the linear regression line through each set of data points (Figure 6C). As indicated by the slope, AZD1152-HQPA increased the turnover rate of Aurora B. To test if the turnover of Aurora B was via the proteasome pathway, an experiment in which MDA-MB-231 cells were treated with or without AZD1152-HQPA in the presence of the proteasome inhibitor, MG132 (Figure 6D) was 


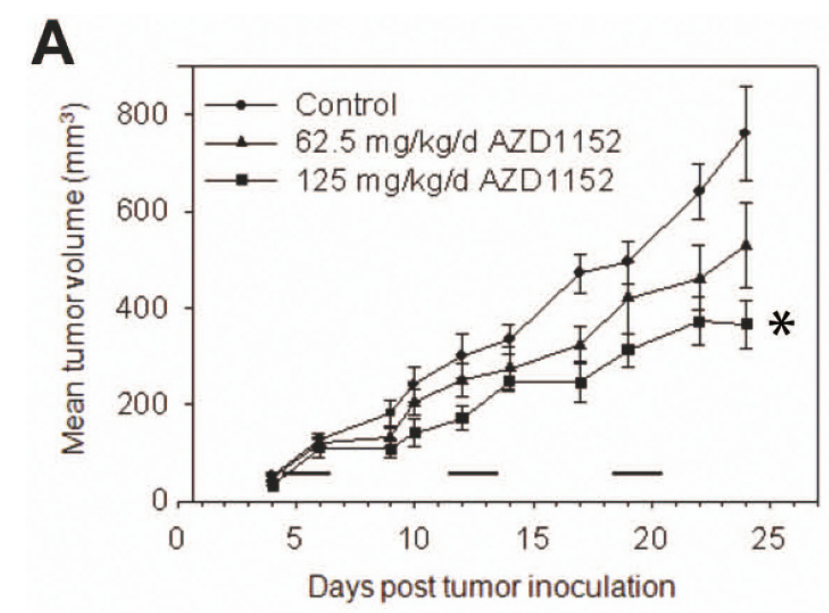

C

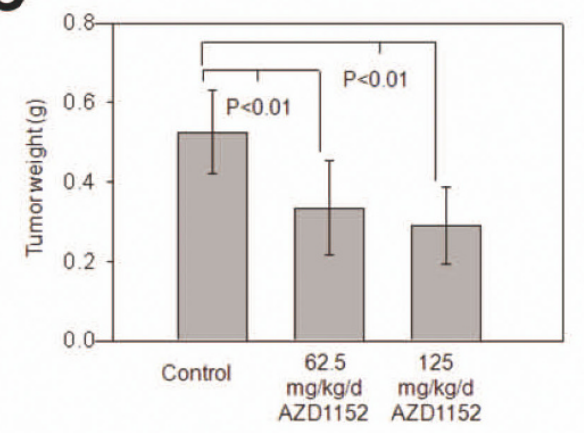

E

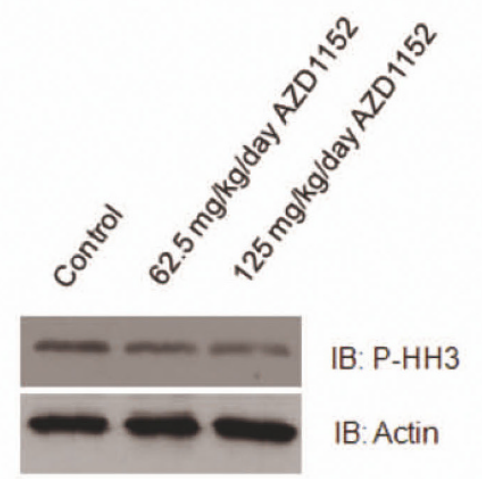

$\mathrm{Ki}-67$
B

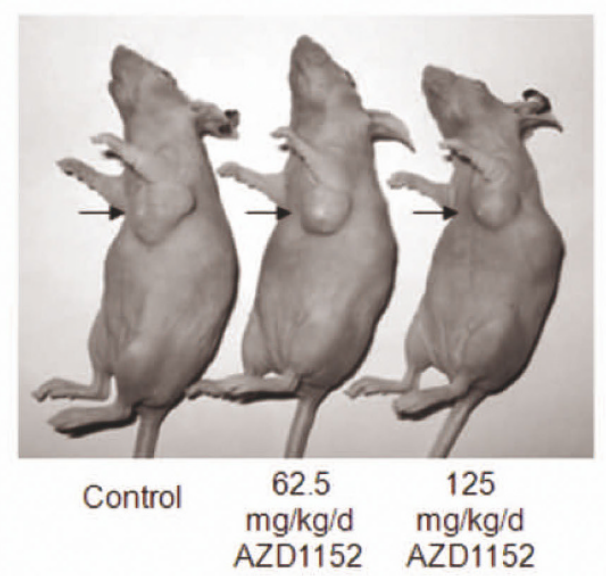

D

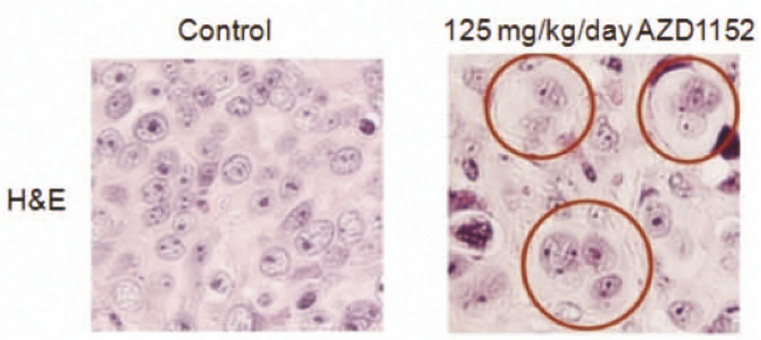

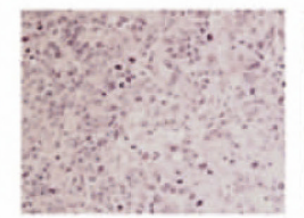
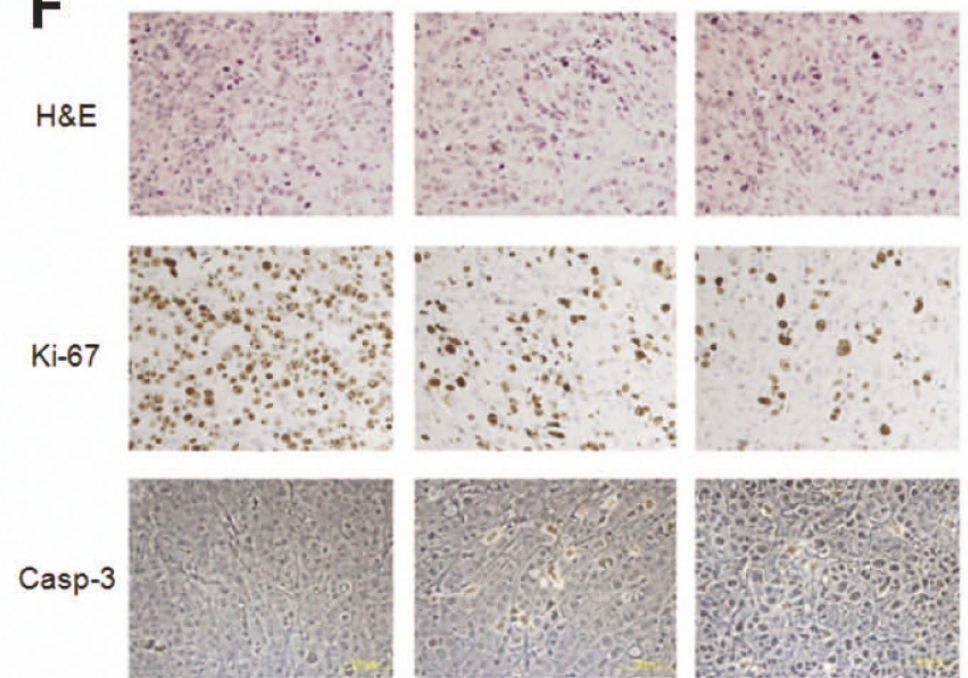

Control

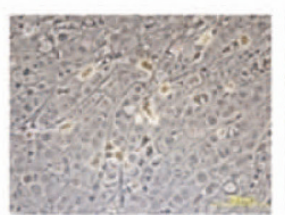

$62.5 \mathrm{mg} / \mathrm{kg} / \mathrm{day}$ AZD1152

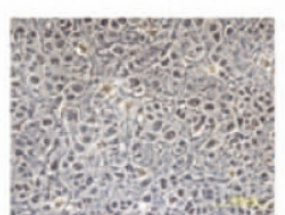

$125 \mathrm{mg} / \mathrm{kg} /$ day AZD1152

Figure 4 AZD1152 inhibits orthotopically xenografted HER18 cells in nude mice. (A) Tumor growth curves for orthotopically implanted HER18 cells are shown. Control mice received vehicle (0.3 M Tris pH 9.0), low dose mice received $62.5 \mathrm{mg} / \mathrm{kg} / \mathrm{dose}$ AZD1152, and high dose mice received $125 \mathrm{mg} / \mathrm{kg} /$ dose AZD1152. Treatment began when tumors were measurable (approximately $50 \mathrm{~mm}^{3}$ ). Injections were given IP on days 1 and 2 of 7-day cycles, indicated by horizontal bars just above the x-axis. ${ }^{*} \mathrm{P}<0.001$ calculated as a mixed linear model. Error bars represent SE. (B) Representative mice bearing xenografts (arrows) from each treatment group. (C) Weights of dissected xenografts are plotted as means for each treatment group. Error bars represent 95\% confidence intervals. P values were determined by one-way ANOVA. (D) H\&E stained control and high dose $(125 \mathrm{mg} / \mathrm{kg} /$ day) tumor samples are shown. Multi-nucleate cells (circles) were observed in tumor sections from AZD1152treated mice but not in control mice. (E) Western blots using anti-phospho-Histone $\mathrm{H} 3$ and anti-actin are shown as labeled. (F) Histology and immunohistochemistry of xenografts are shown: top panels- H\&E staining; middle panels- immunohistochemistry with antibodies for Ki-67; lower panels- cleaved Caspase 3. 

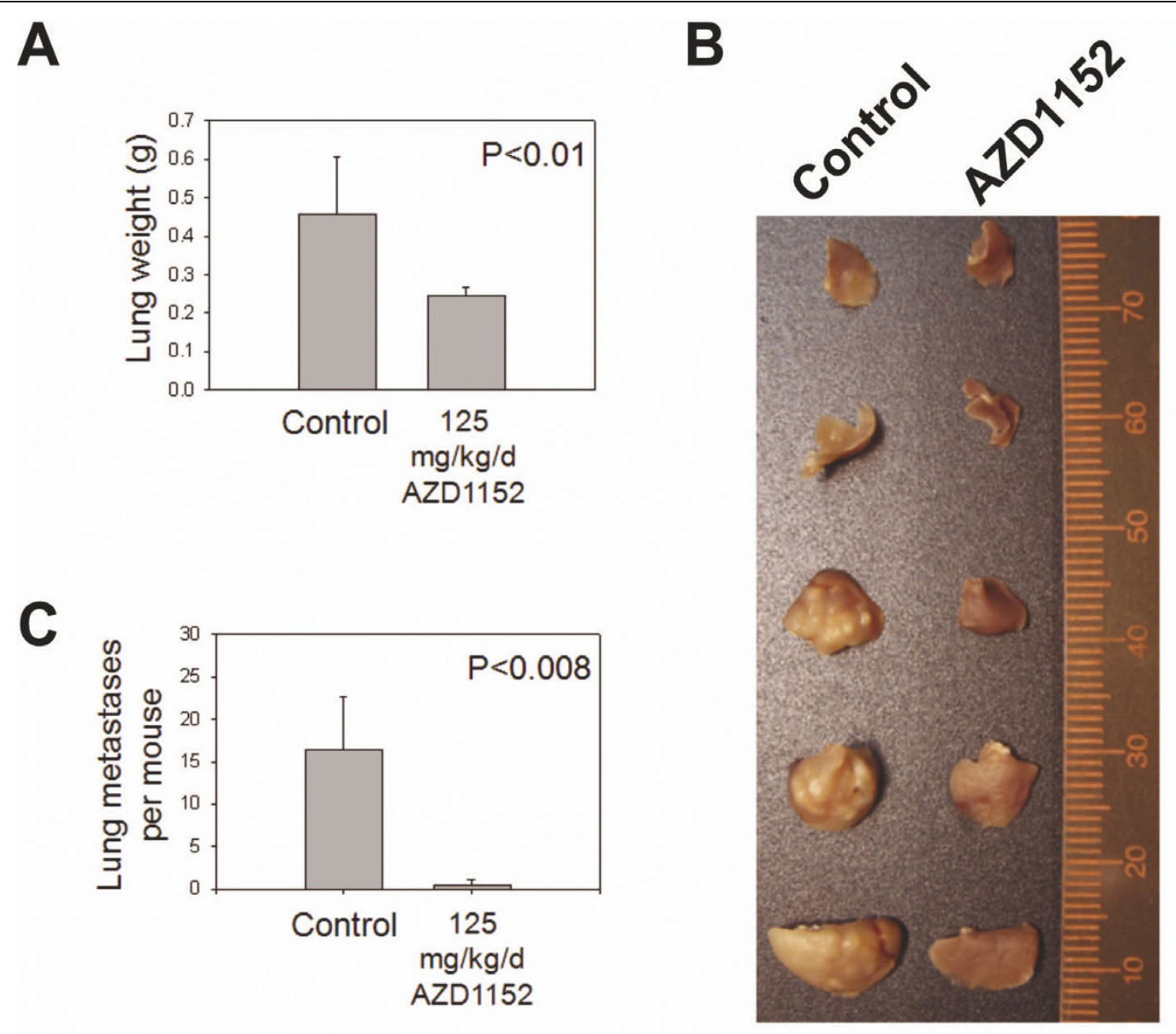

D

Control

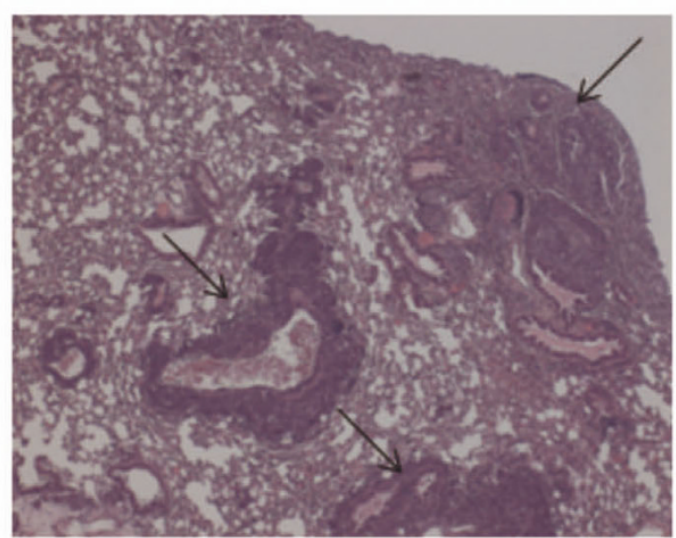

AZD1152

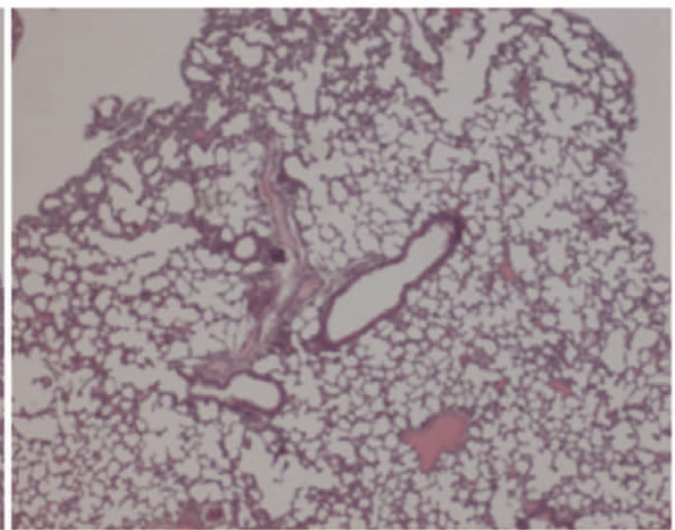

Figure 5 AZD1152 inhibits growth of lung tumor nodules in a lung metastasis nude mouse model. (A) Two million MDA-MB-231 human breast cancer cells were injected via tail veins of nude mice. AZD1152 (125 mg/kg/dose) or vehicle were administered IP on days 1 and 2 of 7 day repeating cycle for 4 cycles starting 2 days after cancer cell injection. Ten weeks after cancer cell injection, the mice were sacrificed and lungs were weighed and plotted as the means for each treatment group. (B) Matched lobes of the lungs from a representative mouse from each group are shown as labeled. (C) The number of macroscopic tumor nodules in the lungs was counted in each mouse. The mean number of nodules per mouse was plotted for each group. Error bars represent 95\% confidence intervals. (D) H\&E stained slides of lungs from control and drug treated mice are shown at the same magnification. Arrows indicate metastatic lung tumors. 


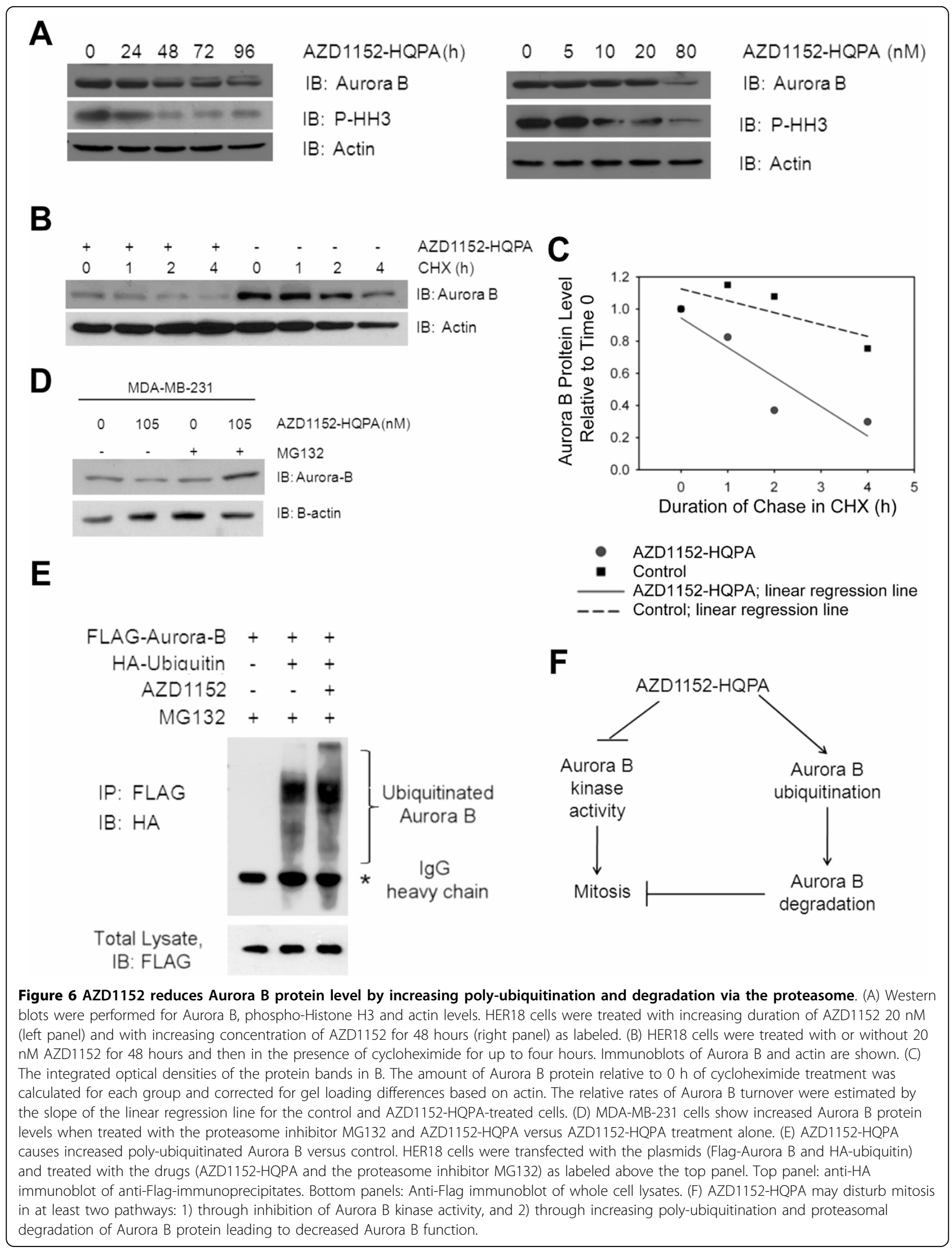


performed. The result shows that Aurora B protein levels were rescued by inhibition of the proteasome in the presence of AZD1152-HQPA.

Next, the ubiquitination of Aurora $B$ in the presence of AZD1152-HQPA was investigated. HER18 cells were transfected with Flag-tagged Aurora B and HA-tagged ubiquitin. The transfected cells were incubated in the presence or absence of $20 \mathrm{nM}$ AZD1152-HQPA. All samples were incubated with MG132 $(10 \mu \mathrm{g} / \mathrm{ml}, 5 \mathrm{~h})$ to inhibit the proteasome. Immunoprecipitation with antiFlag antibody followed by immunoblotting with anti-HA antibody demonstrated the presence of polyubiquitinated Aurora $B$ in cells transfected with both plasmids while no polyubiquitinated Aurora B and only the IgG heavy chain band was detected in cells transfected with Flag-Aurora B only (Figure 6E). Taken together, these results suggest that subsequent to inhibition of Aurora B kinase activity by AZD1152-HQPA, Aurora B protein turnover was increased by polyubiquitination and proteasomal degradation.

\section{Discussion}

In this study, a knowledge gap regarding the use of an Aurora B kinase inhibitor in models of breast cancer was addressed. AZD1152-HQPA has the ability to inhibit a range of human breast cancer cell lines with $\mathrm{IC}_{50} \mathrm{~S}$ in the range of 8-125 $\mathrm{nM}$ (Figure 1), which is similar to the range for leukemia [1] and colorectal cancer [7] cell lines. This concentration range is well below the concentrations where AZD1152-HQPA would inhibit Aurora A or other kinases [1]. Further, these six cell lines have various molecular profiles for HER2, ER, PR and p53. This is a potentially important finding in that this drug may provide an alternative method for treatment of breast cancer regardless of its molecular profile. One of the breast cancer cells lines assayed, MB-MDA 231 (HER2+ [16], ER-, PR-, p53 mutant), is considered to be a "triple negative" form of breast cancer. AZD1152HQPA was also shown to be effective in this line which underscores the potential therapeutic value of this drug in a limited druggable-target form of breast cancer.

While investigating the cellular effects of AZD1152HQPA it was found that it caused mitotic catastrophe resulting in aneuploidy, polyploidy and various other chromosomal abnormalities (Figure 2). Treatment with AZD1152-HQPA in breast cancer cells also led to apoptosis (Figure 3) as previously found in leukemia, multiple myeloma and colorectal cancer cells $[1,7,10]$. Additionally, it was found that colony forming potential in both anchorage dependent and anchorage-independent assays were reduced by this drug. These cell culture data prompted further investigation into the antineoplastic activity of AZD1152 in an in vivo model.
In an orthotopic xenograft model (Figure 4) and a lung metastasis model (Figure 5) of breast cancer, the in vivo antineoplastic effect of AZD1152 is demonstrated. The pharmacodynamic mechanism of inhibition of Aurora B kinase activity is confirmed in vivo by the decrease in phosphorylation of Histone $\mathrm{H} 3$ at serine 10 in xenografts as measured by immunoblotting with phospho-specific antibody. AZD1152 was well tolerated by the mice and no digestive distress or significant weight loss was observed. The in vivo antineoplastic effect demonstrated in these experiments warrants further investigation of this drug in clinical trials for breast cancer.

It has been discovered that in addition to inhibiting Aurora B kinase activity, AZD1152-HQPA also decreases Aurora B protein level in a dose- and timedependent manner (Figure 6A). Mechanistic studies show that AZD1152-HQPA increases the turnover rate of Aurora $B$ by increasing polyubiquitination and proteasomal degradation. The results uncover a previously unknown regulatory circuitry of AZD1152. Until now, no Aurora kinase inhibitor has been reported to affect the protein level of any Aurora kinase. These results also generate new hypotheses. Because Aurora B is autophosphorylated [25], it remains to be determined whether AZD1152 can cause inhibition of autophosphorylation, thereby enhancing the Aurora B degradation. Such a possibility will be another interesting layer of regulation to explore. These findings highlight the complexity of AZD1152's impact on post-translational regulation of Aurora $B$ and provide the important insight that the inhibitory effect of AZD1152-HQPA may persist after wash-out of the drug from the target tissue. Theoretically, the inhibition of Aurora B should persist until the Aurora B protein level returns to normal. Therefore, the biological effects could be expected to last longer than the presence of the active inhibitor AZD1152-HQPA. This novel finding may aid the proper interpretation and use of pharmacokinetic and pharmacodynamic information about this drug to improve design of clinical dosing regimens.

\section{Conclusions}

AZD1152-HQPA has antineoplastic activity against breast cancer cells in culture, and AZD1152 suppressed breast cancer growth in two mouse models. AZD1152HQPA accelerated turnover of Aurora B protein by increasing poly-ubiquitination and proteasomal degradation. The finding that AZD1152-HQPA downregulates Aurora B protein level as well as inhibits Aurora B kinase activity (Figure 6F) will be important for the interpretation of pharmacokinetics and pharmacodynamics of AZD1152. 


\section{Methods \\ Cell lines}

HER18 cells (stably expressing HER2) were described previously [19] and were cultured in DME/F12 media (Sigma, St. Louis, MO) supplemented with 10\% (v/v) fetal bovine serum (Gemini, West Sacramento, CA). Human breast cancer cell lines MDA-MB-231, MDAMB-435, MDA-MB-361, BT-474 and MDA-MB-468 were obtained from ATCC (Manassas, VA, USA). MDA-MB-231, MDA-MB-435 and MDA-MB-468 cells were cultured in Leibovitz's L15 media (Cellgro, Manassas, VA) with $10 \%(\mathrm{v} / \mathrm{v})$ fetal bovine serum, $2 \mathrm{mM} \mathrm{L-}$ glutamine (Cellgro, Manassas, VA) and 1\% (v/v) antibiotic-antimycotic solution (Invitrogen, Grand Island, NY). BT-474 and MDA-MB-361 were cultured in DME/F12 media (Sigma, St. Louis, MO) supplemented with $10 \%$ (v/v) fetal bovine serum (Gemini, West Sacramento, CA). All the cells were incubated at $37^{\circ} \mathrm{C}$ with $5 \% \mathrm{CO}_{2}$.

\section{Compounds and antibodies}

Antibodies used in Western blots include: anti-phosphoHistone H3 (p-HH3) (Upstate, Lake Placid, NY), antiPARP (Cell Signaling, Danvers, MA), anti-Aurora B (Abcam, Cambridge, MA), anti-HA (Sigma) and antiactin (Sigma). The protein synthesis inhibitor cycloheximide and proteasome inhibitor MG132 were purchased from Sigma Chemical Co. (St. Louis, MO).

AZD1152 prodrug and its metabolite, AZD1152HQPA, were provided by Astra Zeneca Pharmaceuticals (Macclesfield, Cheshire, UK). AZD1152-HQPA was dissolved in $100 \%$ DMSO at $10 \mathrm{mM}$, and used at indicated durations and concentrations after dilution in tissue culture media. AZD1152 was dissolved in $0.3 \mathrm{M}$ Tris $\mathrm{pH}$ 9.0 (Fisher, Pittsburgh, PA) and 0.2\% DMSO (Fisher) at a maximum concentration of $20 \mathrm{mg} / \mathrm{ml}$. The AZD1152 solution was made fresh for each round of injection into mice.

\section{MTT (3-(4,5-dimethylthiazol-2-yl)-2,5-diphenyltetrazolium bromide) assay}

Cell lines were seeded at 5-20\% confluence in 96-well microplates and allowed to attach for 24 hours. AZD1152-HQPA was serially diluted in appropriate complete media to the stated final concentrations. Plates were incubated for 2 to 5 days. Following incubation, 20 $\mu \mathrm{l}$ of 3-(4,5-dimethylthiazol-2-yl)-2,5-diphenyltetrazolium bromide (MTT) (Sigma) was added to each well. After incubation for 1 to 5 hours, the media were replaced by $200 \mu \mathrm{l} 100 \%$ DMSO in each well. After mixing, the microplates were read with an MRX revolution plate spectrophotometer (Dynex, Technologies, Chantilly, VA) at $570 \mathrm{~nm}$. Averages of at least 4 replicates were plotted and $50 \%$ inhibitory concentrations $\left(\mathrm{IC}_{50}\right)$ were estimated based on sigmoidal curve fitting. For validation of MTT method in HER18 cells, cells from 6$\mathrm{cm}$-diameter dishes after similar treatment with AZD1152-HQPA were counted using a Z1 Coulter particle counter (Beckman Coulter, Fullerton, CA), and average counts from triplicate plates were plotted against concentration of AZD1152-HQPA.

\section{Evaluation of in vivo antineoplastic activity}

Six - to eight-week old female athymic $(\mathrm{nu} / \mathrm{nu})$ mice (Experimental Radiation Oncology, MD Anderson Cancer Center, Houston, TX, USA) were housed in AAALAC approved barrier facilities on a 12-hour light/dark cycle, with food and water ad libitum. Mice were treated under approved protocols in compliance with the animal care and use guidelines of our institution, the USDA and the NIH. For the breast cancer orthotopic xenograft model, nude mice were injected with $8.5 \times 10^{6}$ HER18 human breast cancer cells in the mammary fat pad $[26,27]$. The nude mice were supplemented with weekly subcutaneous estradiol cypionate (Pfizer, NY, NY) injections (3 mg/kg/week) $[20,21]$ starting 2 weeks prior to injection of tumor cells. Mice were randomized into 3 groups and treatment with AZD1152 prodrug began when tumors were measurable (approximately $50 \mathrm{~mm}^{3}$ ). Control mice received IP injections of vehicle $(0.3 \mathrm{M}$ Tris $\mathrm{pH} 9.0$ ), low dose group received $62.5 \mathrm{mg} / \mathrm{kg} /$ day of AZD1152, and the high dose group received $125 \mathrm{mg} / \mathrm{kg} /$ day of AZD1152 on days 1 and 2 of a 7-day cycle for 3 cycles. Tumor measurements were taken every 2 to 3 days, and volumes were estimated with this formula: Length $\times \mathrm{Width}^{2} / 2$ [28]. Mice were sacrificed 24 days post tumor cell injection, and tumors were dissected. Tumor samples were snap frozen for Western blotting or fixed in formalin and paraffin embedded for histological analysis. Immunohistochemistry (IHC) was performed using the ABC kit (Vector Lab.) according to standard techniques. Photomicrographs were taken at $200 \times$ magnification with an Olympus IX70 microscope and Olympus DP controller imaging software (version. 3.2.1.276).

For the breast cancer lung metastasis model, nude mice were injected with $2 \times 10^{6}$ MDA-MB-231 cells via a tail vein. Cells were suspended in $200 \mu \mathrm{l}$ complete media (DME/F12 with 10\% fetal bovine serum). Mice were randomized into 2 groups (control and AZD1152). The AZD1152 group received injections of AZD1152 $125 \mathrm{mg} / \mathrm{kg} /$ day IP on days 1 and 2 of a 7-day cycle for 4 cycles starting two days after tumor cell injection. Control mice received IP injections of vehicle $(0.3 \mathrm{M}$ Tris $\mathrm{pH}$ 9.0). Mice were sacrificed 10 weeks after tumor cell injection, and the lungs were weighed and examined for tumor nodules. Tumor samples were fixed in formalin and paraffin embedded for histological analysis. 


\section{Flow cytometry}

Breast cancer cells for cell cycle analysis were plated in 100-mm tissue culture dishes and allowed to attach for 24 hours. $100 \mathrm{nM}$ AZD1152-HQPA or vehicle were applied to each plate for indicated times and cells harvested by trypsinization (Cellgro, Herndon, VA). Cells were collected and fixed in $70 \%$ ethanol for 1 hour followed by additional washing in PBS pH 7.40. Propidium iodide (PI) solution $(50 \mu \mathrm{g} / \mathrm{ml})$ with RNAseA $(20 \mu \mathrm{g} / \mathrm{ml})$ (Qiagen, Valencia, CA) was added to each sample and samples analyzed with a FACScalibur flow cytometer (Becton Dickinson, Franklin Lakes, NJ, USA). Cells analyzed for apoptosis were plated in 100-mm dishes and allowed to attach for 24 hours before addition of AZD1152-HQPA. Cells were washed with PBS and trypsinized at appropriate times for harvest. Cells were suspended in $0.5 \mathrm{ml}$ binding buffer (Axxora, San Diego, $\mathrm{CA}$ ) and $5 \mu \mathrm{l}$ Annexin V-FITC (BD, San Jose, CA) for 15 minutes at room temperature in the dark. The cells were washed again, and PI solution with RNAseA was applied just before analysis.

\section{Colony forming assays}

Soft agar colony forming assays were performed in triplicate plates with a base agar of $1 \times$ DME/F12 complete medium with $0.5 \%$ low melt agar (Fisher) and either DMSO for the control or $80 \mathrm{nM}$ AZD1152-HQPA. Base agar was added to $6 \mathrm{~cm}$ Petri dishes and allowed to set. 5000 cells/plate added to $0.35 \%$ top agar with control medium or $80 \mathrm{nM}$ AZD1152-HQPA. The plates were incubated at $37^{\circ} \mathrm{C}$ with $5 \% \mathrm{CO}_{2}$ for 26 days. Colonies $>100$ cells were counted using a dissecting microscope, and results were analyzed using the Student's t-test.

Colony forming assays were performed in triplicate by trypsinizing breast cancer cells and plating them in 6$\mathrm{cm}$ tissue culture dishes at 5000 cells/plate in complete DME/F12 medium with either DMSO or $40 \mathrm{nM}$ AZD1152-HQPA. The plates were incubated for 12 days at $37^{\circ} \mathrm{C}$ with $5 \% \mathrm{CO}_{2}$ for 12 days. Colonies were counted in 3 representative one $\mathrm{cm}^{2}$ areas/plate and the number colonies per $\mathrm{cm}^{2}$ between AZD1152-HQPAtreated cells and control cells were compared using Student's t-test.

\section{Fluorescence microscopy}

Breast cancer cells were grown in chamber slides and treated with either DMSO or $20 \mathrm{nM}$ AZD1152-HQPA for 48 hours. The chamber slides were rinsed $2 \times$ with cold PBS solution and fixed with $10 \%$ formaldehyde (Sigma). Cells were permeabilized with $0.2 \%$ Triton X100 (Sigma), stained with DAPI (Invitrogen) and visualized with an Olympus IX81 fluorescent microscope.

\section{Acknowledgements}

We thank Dr. K Mundt and Dr. E Anderson of Astra Zeneca Pharmaceuticals for providing AZD1152 (prodrug) and AZD1152-HQPA. This study was supported by grants from the National Institutes of Health 5P30CA016672-29 and National Cancer Institute CA16672, CA089266. CG is the recipient of an $\mathrm{NIH}$ training grant fellowship T32 CA009299. GVM is supported with a cancer prevention fellowship by the National Cancer Institute grant R25T CA57730. Additional thanks to C-H Su, R Zhao, Y-S Feng, B Chen, and L Phan for technical support and to SWG for editing.

\section{Author details}

'Department of Molecular and Cellular Oncology, The University of Texas MD Anderson Cancer Center, Houston, TX 77030, USA. ${ }^{2}$ The University of Texas Graduate School of Biomedical Sciences at Houston, Houston, TX 77030, USA. ${ }^{3}$ The University of Texas MD Anderson Cancer Center, Program in Genes and Development, Houston, TX 77330, USA. ${ }^{4}$ Department of General Internal Medicine, Ambulatory Treatment \& Emergency Care, The University of Texas MD Anderson Cancer Center, Houston, TX 77030, USA. ${ }^{5}$ Department of Endocrine Neoplasia and Hormonal Disorders, The University of Texas MD Anderson Cancer Center, Houston, TX 77030, USA. ${ }^{6}$ The University of Texas MD Anderson Cancer Center, Program in Cancer Biology, Houston, TX 77330, USA.

\section{Authors' contributions}

Experiments were performed by CG, FZ, JC, JY, GVT, and EW. CG, SCY and $\mathrm{MHL}$ conceived the experimental design. All authors approved the final manuscript.

\section{Competing interests}

The authors declare that they have no competing interests.

Received: 20 October 2009

Accepted: 22 February 2010 Published: 22 February 2010

\section{References}

1. Yang J, Ikezoe T, Nishioka C, Tasaka T, Taniguchi A, Kuwayama Y, Komatsu N, Bandobashi K, Togitani K, Koeffler HP, et al: AZD1152, a novel and selective aurora B kinase inhibitor, induces growth arrest, apoptosis, and sensitization for tubulin depolymerizing agent or topoisomerase II inhibitor in human acute leukemia cells in vitro and in vivo. Blood 2007, 110:2034-2040.

2. Tao Y, Zhang P, Girdler F, Frascogna V, Castedo M, Bourhis J, Kroemer G, Deutsch $E$ : Enhancement of radiation response in p53-deficient cancer cells by the Aurora-B kinase inhibitor AZD1152. Oncogene 2008, 27:3244-3255.

3. Katayama $H$, Brinkley WR, Sen S: The Aurora kinases: role in cell transformation and tumorigenesis. Cancer Metastasis Rev 2003, 22:451-464.

4. Fu J, Bian M, Jiang Q, Zhang C: Roles of Aurora kinases in mitosis and tumorigenesis. Mol Cancer Res 2007, 5:1-10.

5. Ducat $D$, Zheng Y: Aurora kinases in spindle assembly and chromosome segregation. Exp Cell Res 2004, 301:60-67.

6. Lens SM, Medema RH: The survivin/Aurora B complex: its role in coordinating tension and attachment. Cell Cycle 2003, 2:507-510.

7. Wilkinson RW, Odedra R, Heaton SP, Wedge SR, Keen NJ, Crafter C, Foster JR, Brady MC, Bigley A, Brown E, et al: AZD1152, a selective inhibitor of Aurora B kinase, inhibits human tumor xenograft growth by inducing apoptosis. Clin Cancer Res 2007, 13:3682-3688.

8. Hsu JY, Sun ZW, Li X, Reuben M, Tatchell K, Bishop DK, Grushcow JM, Brame CJ, Caldwell JA, Hunt DF, et al: Mitotic phosphorylation of histone $\mathrm{H} 3$ is governed by Ipl1/aurora kinase and Glc7/PP1 phosphatase in budding yeast and nematodes. Cell 2000, 102:279-291.

9. Vader G, Cruijsen CW, van Harn T, Vromans MJ, Medema RH, Lens SM: The chromosomal passenger complex controls spindle checkpoint function independent from its role in correcting microtubule kinetochore interactions. Mol Biol Cell 2007, 18:4553-4564. 
10. Evans RP, Naber C, Steffler T, Checkland T, Maxwell CA, Keats JJ, Belch AR, Pilarski LM, Lai $R$, Reiman $T$ : The selective Aurora $B$ kinase inhibitor AZD1152 is a potential new treatment for multiple myeloma. $\mathrm{Br} J$ Haematol 2008, 140:295-302.

11. Ditchfield C, Johnson VL, Tighe A, Ellston R, Haworth C, Johnson T, Mortlock A, Keen N, Taylor SS: Aurora B couples chromosome alignment with anaphase by targeting BubR1, Mad2, and Cenp-E to kinetochores. J Cell Biol 2003, 161:267-280.

12. Keen N, Taylor S: Aurora-kinase inhibitors as anticancer agents. Nat Rev Cancer 2004, 4:927-936.

13. Tanaka T, Kimura M, Matsunaga K, Fukada D, Mori H, Okano Y: Centrosomal kinase AIK1 is overexpressed in invasive ductal carcinoma of the breast. Cancer Res 1999, 59:2041-2044.

14. Nadler Y, Camp RL, Schwartz C, Rimm DL, Kluger HM, Kluger Y: Expression of Aurora A (but not Aurora B) is predictive of survival in breast cancer. Clin Cancer Res 2008, 14:4455-4462.

15. Cochran AG: Aurora A: target invalidated?. Chem Biol 2008, 15:525-526.

16. Bischoff JR, Anderson L, Zhu Y, Mossie K, Ng L, Souza B, Schryver B, Flanagan P, Clairvoyant F, Ginther $C$, et al: A homologue of Drosophila aurora kinase is oncogenic and amplified in human colorectal cancers. Embo J 1998, 17:3052-3065.

17. Girdler F, Sessa F, Patercoli S, Villa F, Musacchio A, Taylor S: Molecular basis of drug resistance in aurora kinases. Chem Biol 2008, 15:552-562.

18. Mortlock AA, Foote KM, Heron NM, Jung FH, Pasquet G, Lohmann JJ, Warin N, Renaud F, De Savi C, Roberts NJ, et al: Discovery, synthesis, and in vivo activity of a new class of pyrazoloquinazolines as selective inhibitors of aurora B kinase. J Med Chem 2007, 50:2213-2224.

19. Laronga C, Yang HY, Neal C, Lee MH: Association of the cyclin-dependent kinases and 14-3-3 sigma negatively regulates cell cycle progression. J Biol Chem 2000, 275:23106-23112.

20. Jerome L, Alami N, Belanger S, Page V, Yu Q, Paterson J, Shiry L, Pegram M, Leyland-Jones B: Recombinant human insulin-like growth factor binding protein 3 inhibits growth of human epidermal growth factor receptor-2overexpressing breast tumors and potentiates herceptin activity in vivo. Cancer Res 2006, 66:7245-7252.

21. Wang LH, Yang XY, Zhang X, Mihalic K, Fan YX, Xiao W, Howard OM, Appella E, Maynard AT, Farrar WL: Suppression of breast cancer by chemical modulation of vulnerable zinc fingers in estrogen receptor. Nat Med 2004, 10:40-47.

22. Goto $H$, Yasui $Y$, Nigg EA, Inagaki M: Aurora-B phosphorylates Histone H3 at serine28 with regard to the mitotic chromosome condensation. Genes Cells 2002, 7:11-17.

23. Wei $Y$, Yu L, Bowen J, Gorovsky MA, Allis CD: Phosphorylation of histone $\mathrm{H} 3$ is required for proper chromosome condensation and segregation. Cell 1999, 97:99-109.

24. Hirota T, Lipp JJ, Toh BH, Peters JM: Histone $\mathrm{H} 3$ serine 10 phosphorylation by Aurora B causes HP1 dissociation from heterochromatin. Nature 2005, 438:1176-1180.

25. Yasui Y, Urano T, Kawajiri A, Nagata K, Tatsuka M, Saya H, Furukawa K, Takahashi T, Izawa I, Inagaki M: Autophosphorylation of a newly identified site of Aurora-B is indispensable for cytokinesis. J Biol Chem 2004, 279:12997-13003.

26. McKenzie T, Liu Y, Fanale M, Swisher SG, Chada S, Hunt KK: Combination therapy of Ad-mda7 and trastuzumab increases cell death in Her-2/neuoverexpressing breast cancer cells. Surgery 2004, 136:437-442.

27. Warburton C, Dragowska WH, Gelmon K, Chia S, Yan H, Masin D, Denyssevych T, Wallis AE, Bally MB: Treatment of HER-2/neu overexpressing breast cancer xenograft models with trastuzumab (Herceptin) and gefitinib (ZD1839): drug combination effects on tumor growth, HER-2/neu and epidermal growth factor receptor expression, and viable hypoxic cell fraction. Clin Cancer Res 2004, 10:2512-2524.

28. Osborne CK, Hobbs K, Clark GM: Effect of estrogens and antiestrogens on growth of human breast cancer cells in athymic nude mice. Cancer Res $1985,45: 584-590$.

doi:10.1186/1476-4598-9-42

Cite this article as: Gully et al:: Antineoplastic effects of an Aurora B kinase inhibitor in breast cancer. Molecular Cancer 2010 9:42.

\section{Submit your next manuscript to BioMed Central and take full advantage of:}

- Convenient online submission

- Thorough peer review

- No space constraints or color figure charges

- Immediate publication on acceptance

- Inclusion in PubMed, CAS, Scopus and Google Scholar

- Research which is freely available for redistribution

Submit your manuscript at www.biomedcentral.com/submit 